\title{
A variational-based fusion model for non-uniform illumination image enhancement via contrast optimization and color correction
}

\author{
Qi-Chong Tian*, Laurent D. Cohen* \\ CEREMADE, CNRS, Université Paris-Dauphine, PSL Research University, Paris 75016, France
}

\section{A R T I C L E I N F O}

\section{Article history:}

Received 17 February 2018

Revised 26 July 2018

Accepted 27 July 2018

Available online 29 July 2018

\section{Keywords:}

Image enhancement

Contrast optimization

Color correction

Image fusion

Variational methods

\begin{abstract}
A B S T R A C T
Non-uniform illumination images are of limited visibility due to under-exposure, over-exposure, or a combination of these two factors. Enhancing these images is a very challenging task in image processing. Although there are numerous enhancement methods to improve the visual quality of images, many of these methods produce undesirable results with regard to contrast and saturation improvements. In order to improve the visibility of images without over-enhancement or under-enhancement, a variationalbased fusion method is proposed for adaptively enhancing non-uniform illumination images. First, a huepreserving global contrast adaptive enhancement algorithm obtains a globally enhanced image. Second, a hue-preserving local contrast adaptive enhancement method produces a locally enhanced image. Finally, an enhanced result is obtained by a variational-based fusion model with contrast optimization and color correction. The final result represents a trade-off between global contrast and local contrast, and also maintains the color balance between the globally enhanced image and the locally enhanced image. This method produces visually desirable images in terms of contrast and saturation improvements. Experiments were conducted on a dataset that included different kinds of non-uniform illumination images. The results demonstrate that the proposed method outperforms the compared enhancement algorithms both qualitatively and quantitatively.
\end{abstract}

(c) 2018 Elsevier B.V. All rights reserved.

\section{Introduction}

The advent of digital cameras makes it very convenient to obtain images. However, some images contain poor visual details, which results from the limitations of imaging devices or environmental illuminations. To improve the visibility of images, many enhancement methods [1-8] have been proposed. Enhancement methods are able to improve the visual quality of images, so they have been widely applied to various fields of imaging, such as photography [9], medical imaging [10], and remote-sensing imaging [11]. Since image enhancement methods can improve the visibility of images, they are often used for pre-processing images in some other computer vision applications and image processing problems, including object segmentation [12], face recognition [13], and high dynamic range image rendering [14]. Moreover, these kinds of image enhancement methods are also applied in post-processing images and videos.

\footnotetext{
* Corresponding authors.

E-mail addresses:

cohen@ceremade.dauphine.fr (LD. Coheremade.dauphine.fr

URL: https://www.ceremade.dauphine.fr/ cohen (L.D. Cohen)
}

Images captured in uniform illumination environments can be enhanced well in terms of contrast improvements. However, most of the enhancement algorithms fail in processing non-uniform illumination images. Some existing methods [1-3,5,8,15] either underenhance dark regions or over-enhance bright regions of images. Generally speaking, enhancement methods are categorized into two types: global enhancement algorithms and local enhancement algorithms. Usually, global enhancement methods produce overenhanced results in the bright regions of images, while local enhancement algorithms obtain under-enhanced results in the dark regions of images. An example is shown in Fig. 1, from which we can see that the global enhancement method Histogram Equalization (HE) [15] over-enhances the bright regions and the local method Contrast-Limited Adaptive Histogram Equalization (CLAHE) [16] under-enhances the dark regions.

Inspired by the idea of exposure fusion [17], Tian and Cohen [18] proposed an enhancement method that combines globally enhanced images and locally enhanced images. This method improves the contrast both in dark regions and bright regions of images. Following the enhancement framework in [18], we consider a variational method [19] in the fusion model to improve more visibility of images. Specifically, a hue-preserving global contrast adaptive enhancement method is applied to the original non-uniform 


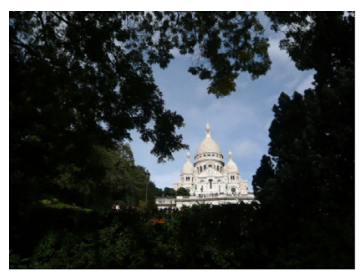

(a) Original

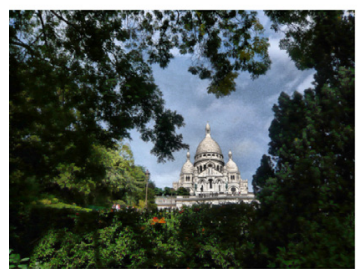

(c) CLAHE

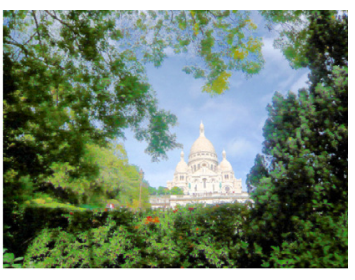

(b) $\mathrm{HE}$

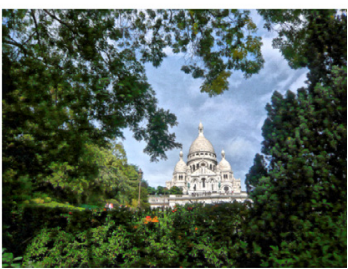

(d) Proposed
Fig. 1. Enhancement results by the global method Histogram Equalization (HE), local method Contrast-Limited Adaptive Histogram Equalization (CLAHE), and the proposed method. The bright regions (white building) are over-enhanced by HE. The dark regions (under-exposed tree) are under-enhanced by CLAHE. The bright regions and dark regions are both enhanced well by the proposed method.

illumination color images. Then, a hue-preserving local contrast adaptive enhancement method is applied to the original images. Finally, the enhanced results are obtained via a variational-based fusion algorithm considering contrast optimization [20,21] and color correction $[22,23]$.

The main contributions of this paper include presenting an adaptive global contrast enhancement method, considering hue preservation in the enhancement framework, and developing a variational-based fusion model via contrast optimization and color correction.

This paper is an extended version of our previous work [18]. Compared with [18], a variational-based fusion model considering contrast optimization and color correction is proposed in this work. The proposed variational-based fusion method produces better results than [18] in terms of contrast improvement. The rest of this paper is organized as follows. The related work is briefly summarized in Section 2. The detailed description of the proposed method is presented in Section 3. Numerical experiments and performance evaluations are shown in Section 4. Finally, we give the conclusions and the future work in Section 5.

\section{Related work}

The proposed image enhancement method combines the results of a global enhancement method and a local enhancement method, with a variational-based image fusion framework. In this section, we will introduce some related techniques, including global enhancement methods, local enhancement methods, and image fusion models.

\subsection{Global enhancement methods}

Global image enhancement methods conduct the same operation on the same pixel value regardless of the corresponding neighboring pixel distribution. The simplest method is a piece-wise linear transformation [15], which defines a poly-line as the mapping function. In order to obtain more visibility of images, some global enhancement methods use various curves to map the considered images. For example, power-law functions, log functions, and gamma functions are often used for non-linear mappings, which produce better results than linear mappings. Global histograms of images can also be used for enhancements. Histogram Equalization (HE) [15] is a widely used technique for image contrast enhancement. HE modifies the original histograms of images to an approximate uniform histogram, which stretches pixel value range of images. The traditional HE might produce over-enhanced results. To avoid this problem, some improved histogram equalization methods are proposed. Arici et al. [5] proposed a general histogram equalization framework for image contrast enhancement via optimizing cost functions. Tian and Cohen [24] presented a naturalness preservation histogram-based enhancement method using structure measure and statistical naturalness measure.

\subsection{Local enhancement methods}

Contrast-Limited Adaptive Histogram Equalization (CLAHE) [16] is another traditional histogram-based enhancement algorithm. CLAHE considers histogram equalization in each local region. This kind of local enhancement methods [25-28] can obtain more details of images, and preserve the image naturalness. Inspired by the retinex theory [29], there are many retinex-based contrast enhancement algorithms. Considering a Gaussian kernel to build the relationship between the center pixel and surrounding pixels, Jobson et al. [30] proposed a classic retinex-based enhancement method. Recently, Petro et al. [31] improved the multiscale retinex model for image enhancement. In order to obtain better visual quality, Kimmel et al. [32] introduced a variational model to the retinex enhancement via a quadratic programming optimization. Moreover, Morel et al. [33] explored some new alternative kernels for the retinex model, which are suitable for different kinds of enhancement applications. Banić and Lončarić [6] proposed a fast retinex implementation for brightness adjustment and color correction, which avoids some disadvantages of traditional retinex enhancement methods. Provenzi et al. [4] combined color correction and local contrast improvement for color image enhancement. Palma-Amestoy et al. [20] proposed a variational framework for color image enhancement, which was inspired by the basic phenomenology of color perception. Recently, Pierre et al. [21] proposed a hue-preserving variational contrast enhancement method, which enables users to control the contrast improvement levels.

\subsection{Image fusion}

Image fusion is a technique of combining useful information from different images, that describe the same scene. The fused result contains better visibility than any of the input images. The fusion can be conducted on various levels, such as signal-level, pixellevel, feature-level, and symbol-level [34]. No matter which level is selected, the fusion result should meet the following two conditions: (1) the fused image preserves as much information as possible from the input images; (2) the fused image does not contain new artifacts or noise. Mertens et al. [17] proposed a practical pixel-level exposure fusion method for generating high dynamic range images. The result maintains useful information in each image sequence by using weighted blending. Ma et al. [35] proposed a feature-level robust exposure fusion model for avoiding ghosting effects. Wang et al. [36] proposed a variational method for fusing multi-focus images. A family of weight functions using the local average modulus of gradients and the power transform was adopted in Wang et al.'s method. Fu et al. [37] proposed a fusion method for combining three illumination components. This method can obtain good results for weakly-illuminated images. Inspired by the human visual system, Ying et al. [38] proposed a multi-exposure fusion method for low-light image enhancement, which produced enhanced results with small lightness distortion. 


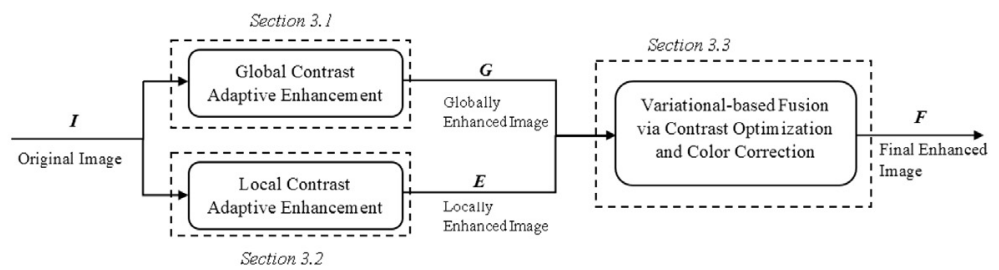

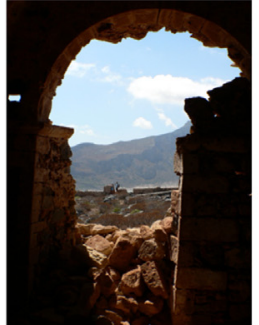

Original Image $I$

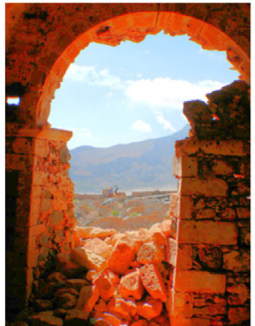

Globally Enhanced Image $\boldsymbol{G}$

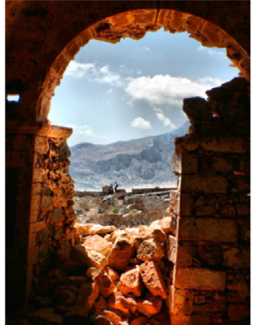

Locally Enhanced Image $E$

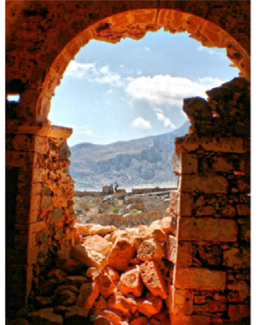

Final Enhanced Image $F$

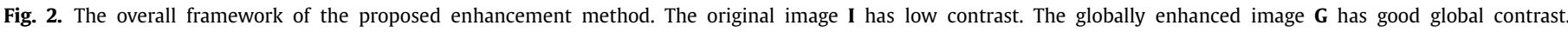

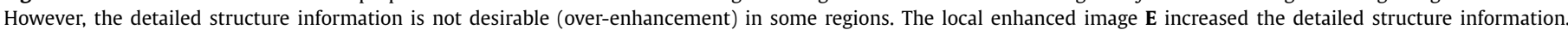
However, the visual quality is not desirable (under-enhancement). The final enhanced image $\mathbf{F}$ represents a trade-off between the global contrast and the local contrast.

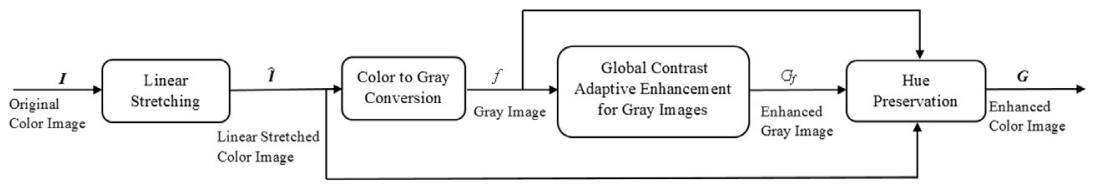

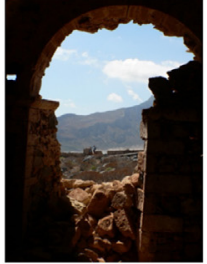

Original Color Image

$I$

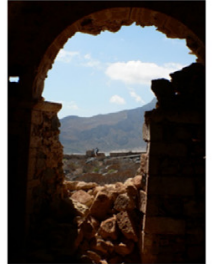

Linear Stretched Color Image

$\hat{I}$

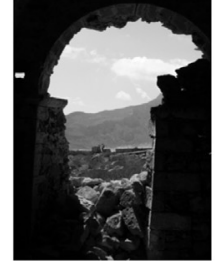

Gray Image

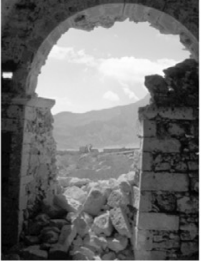

Enhanced Gray Image

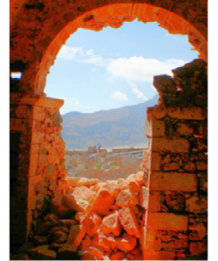

Enhanced Color Image

$G$

Fig. 3. The overview of the global contrast adaptive enhancement method.

\section{The proposed method}

Let $\mathbf{I}=\left(I_{r}, I_{g}, I_{b}\right): \Omega \rightarrow[0,255]^{3}$ be the considered RGB color image, where $\Omega \subset \mathbb{R}^{2}$ is the image domain. $\mathbf{x}=\left(x_{1}, x_{2}\right)$ denotes the position in the image domain $\Omega$. Our goal is to obtain the enhanced color image $\mathbf{F}=\left(F_{r}, F_{g}, F_{b}\right)$. The overall framework of the proposed variational-based fusion method for non-uniform illumination image enhancement via contrast optimization and color correction is illustrated in Fig. 2. The considered non-uniform illumination color image $\mathbf{I}$ is processed by a global enhancement method and a local enhancement method, respectively. Then the globally enhanced color image $\mathbf{G}$ and the locally enhanced color image $\mathbf{E}$ are fused to produce a well-enhanced color image $\mathbf{F}$. The detailed description of the proposed method is presented below.

\subsection{Global contrast adaptive enhancement}

\subsubsection{Overview}

The overall framework of the global contrast adaptive enhancement method is shown in Fig. 3. The brief introduction is given below.

In our global contrast adaptive enhancement method, the pixel value range of a color image I is first converted to the full range $[0,255]$ with the linear stretching [39] via

$\hat{\mathbf{I}}=255 \cdot \frac{\mathbf{I}-I_{\min }}{I_{\max }-I_{\min }}$, where $I_{\min }$ and $I_{\max }$ are the minimal and the maximal intensity values among the three color channels of the image $\mathbf{I}=\left(I_{r}, I_{g}, I_{b}\right)$. $\hat{\mathbf{I}}=\left(\hat{I}_{r} \hat{I}_{g}, \hat{I}_{b}\right)$ is the stretched color image.

The stretched color image $\hat{\mathbf{I}}$ is converted to the corresponding intensity image $f$ using

$f=0.299 \cdot \hat{I}_{r}+0.587 \cdot \hat{I}_{g}+0.114 \cdot \hat{I}_{b}$,

where $\hat{I}_{r}, \hat{I}_{g}$, and $\hat{I}_{b}$ are the three color channels of the stretched color image $\hat{\mathbf{I}}$.

Then, a global contrast adaptive enhancement is conducted on $f$ to obtain the corresponding enhanced intensity image $G_{f}$. The detailed description of this global enhancement algorithm is given in Section 3.1.2. Last, we use the hue preservation enhancement framework to achieve the enhanced color image $\mathbf{G}$ with

$\mathbf{G}(\mathbf{x})= \begin{cases}\frac{G_{f}(\mathbf{x})}{f(\mathbf{x})} \hat{\mathbf{I}}(\mathbf{x}), & \text { if } \frac{G_{f}(\mathbf{x})}{f(\mathbf{x})}<=1, \\ \frac{255-G_{f}(\mathbf{x})}{255-f(\mathbf{x})}(\hat{\mathbf{I}}(\mathbf{x})-f(\mathbf{x}))+G_{f}(\mathbf{x}), & \text { if } \frac{G_{f}(\mathbf{x})}{f(\mathbf{x})}>1,\end{cases}$

where $\mathbf{G}(\mathbf{x})=\left(G_{r}(\mathbf{x}), G_{g}(\mathbf{x}), G_{b}(\mathbf{x})\right)$ represents the globally enhanced color image, $G_{r}(\mathbf{x}), G_{g}(\mathbf{x}), G_{b}(\mathbf{x})$ are the three color channels of $\mathbf{G}$, and $\mathbf{x}$ denotes the positions of pixels on the image domain. Nikolova et al. [40] have analyzed and proven the efficiency of hue preservation with this equation. The hue-preserving enhancement framework is also analyzed in [3], which demonstrates that this kind of framework is better than the traditional channel-by- 
channel enhancements or methods only enhancing the intensity channel.

\subsubsection{Global contrast adaptive enhancement for gray images}

The overall pipeline of the global contrast adaptive enhancement is given in Section 3.1.1. As mentioned above, we will describe the global contrast adaptive enhancement, which is able to obtain $G_{f}$ from $f$. Given a gray image $f$, with a total number of $N$ pixels and an intensity level range of $[0,255] . h_{f}$ is the normalized histogram of the image $f$.

The traditional histogram equalization (HE) [15] uses the original histogram of the considered image to obtain the mapping function. The mapping function $T$ for the image $f$ is given by

$T(i)=\left\lfloor 255 \cdot \sum_{j=0}^{i} h_{f}(j)+0.5\right\rfloor$,

where $i \in[0,255]$ is the input integer for the mapping function, and \lfloor\rfloor computes the nearest integer of the considered value towards minus infinity.

Histogram equalization tries to create a uniform histogram for enhanced images by considering a cumulative histogram as the corresponding mapping function. This method often produces enhanced images with visual artifacts. To avoid this problem, Arici et al. [5] proposed a general histogram modification framework for contrast enhancement. The modified histogram $h$ should be closer to the normalized uniform histogram $h_{U}$, and the value of the residual $h-h_{f}$ should also be small. The problem of obtaining the optimal modified histogram $\tilde{h}$ is regarded as a bi-criteria optimization problem, which can be formulated as a weighted sum of two terms shown in

$\min _{h}\left(\left\|h-h_{f}\right\|+\lambda|| h-h_{U} \|\right)$,

where $\left\|h-h_{f}\right\|$ is a norm of $h-h_{f}$, $\left\|h-h_{U}\right\|$ is a norm of $h-h_{U}$, and the parameter $\lambda>0$ adjusts the trade-off between the contrast enhancement and the data fidelity. An analytical solution of Eq. (5) will be obtained when the squared sum of the $L_{2}$ norm is adopted. This problem is rewritten in

$\tilde{h}=\underset{h}{\arg \min }\left(\left\|h-h_{f}\right\|_{2}{ }^{2}+\lambda\left\|h-h_{U}\right\|_{2}{ }^{2}\right)$.

This is a quadradic optimization problem, and the solution of Eq. (6) is given by

$\tilde{h}=\left(\frac{1}{1+\lambda}\right) h_{f}+\left(\frac{\lambda}{1+\lambda}\right) h_{U}$.

The parameter $\lambda$ needs to be carefully selected to obtain satisfactory enhancement results. Different values of $\lambda$ can produce corresponding modified histograms, thus generating different enhanced images.

In [5], the authors manually gave the parameter $\lambda$. In order to develop an adaptive enhancement method, we adopt the tone distortion of the mapping function $T$ to guide the optimization. The tone distortion measure [41] is defined by

$D(T)=\max _{0 \leq j \leq i \leq 255}\left\{i-j ; T(i)=T(j), h_{f}(i)>0, h_{f}(j)>0\right\}$.

We are able to know that the smaller the tone distortion $D(T)$ is, the smoother the tone is reproduced by the mapping function $T$, from the definition in Eq. (8). The smoother tone means less unnatural-looking in the results. In other words, a small tone distortion is able to avoid unnatural artifacts. The tone distortion measure $D$ is obtained from the mapping function $T$, which is computed with the optimally modified histogram $\tilde{h}$. And the histogram $\tilde{h}$ depends on the weighted parameter $\lambda$. So we can use the tone distortion to select the optimal weighted parameter $\lambda$, which produces an optimally modified histogram and the final mapping function. This mapping function is utilized to produce the globally enhanced image $G_{f}$.

\subsection{Local contrast adaptive enhancement}

The proposed global contrast adaptive enhancement method can improve the global contrast and brightness of the whole image. However, it is essentially a global method, which may reduce the local contrast or the detail information in the original image. So we will employ a local contrast adaptive enhancement to improve the local contrast and preserve the detail information.

In this method, we combine the hue preservation enhancement framework described in Section 3.1.1 and the Contrast-Limited Adaptive Histogram Equalization method (CLAHE) [16] to improve the local contrast and preserve the hue of the images. The locally enhanced gray image $E_{f}$ is obtained using CLAHE. Then, we can produce the locally enhanced color image $E$ via Eq. (9).

$\mathbf{E}(\mathbf{x})= \begin{cases}\frac{E_{f}(\mathbf{x})}{f(\mathbf{x})} \hat{\mathbf{I}}(\mathbf{x}), & \text { if } \frac{E_{f}(\mathbf{x})}{f(\mathbf{x})}<=1, \\ \frac{255-E_{f}(\mathbf{x})}{255-f(\mathbf{x})}(\hat{\mathbf{I}}(\mathbf{x})-f(\mathbf{x}))+E_{f}(\mathbf{x}), & \text { if } \frac{E_{f}(\mathbf{x})}{f(\mathbf{x})}>1,\end{cases}$

where $\mathbf{E}(\mathbf{x})=\left(E_{r}(\mathbf{x}), E_{g}(\mathbf{x}), E_{b}(\mathbf{x})\right)$ represents the locally enhanced color image, $E_{r}(\mathbf{x}), E_{g}(\mathbf{x}), E_{b}(\mathbf{x})$ are the three color channels of $\mathbf{E}$, and $\mathbf{x}$ denotes the positions of pixels on the image domain.

The overall framework of this method is shown in Fig. 4. This framework is similar to the framework shown in Fig. 3. The only difference is that the step Contrast-Limited Adaptive Histogram Equalization takes the place of the step Global Contrast Adaptive for Gray Images. The detailed description of the Contrast-Limited Adaptive Histogram Equalization method (CLAHE) can be found in the paper [16]. In our method, we adopted the Matlab Image Processing Function adapthisteq to implement CLAHE. All parameters of CLAHE are the defaults. The number of rectangular contextual regions is $8 \times 8$. ClipLimt is a contrast factor that prevents over-enhancement of the image, specifically in homogeneous regions. It is equal to 0.01 with the default setting. NBins is equal to 256 , which results in greater dynamic range. Range is set to full, which means the full range $[0,255]$ is used for enhanced images.

\subsection{Variational-based fusion via contrast optimization and color correction}

Inspired by the idea of exposure fusion [17] and variationalbased enhancement [20], we developed a variational-based fusion method considering contrast optimization and color correction to obtain the final enhanced image $\mathbf{F}$ with the globally enhanced image $\mathbf{G}$ and the locally enhanced image $\mathbf{E}$. This fusion framework achieves desirable contrast and saturation for each pixel, since we adopt pixel-level weights to fuse every pixel via contrast optimization and color correction.

\subsubsection{Variational-based fusion model}

The goal is to fuse the globally enhanced image $\mathbf{G}$ and the locally enhanced image $\mathbf{E}$ to create a final enhancement result $\mathbf{F}$, which achieves the optimal contrast and maintains the color balance. Our model operates in the three color channel respectively. Due to the hue-preserving framework analyzed in Section 3.1.1, we only compute the weight maps for the intensity images of the globally enhanced color image and the locally enhanced color image. We then apply the same weight maps to the three color channels. In the following section, we give the variational-based fusion model for the gray-scale images corresponding to the color channels. 


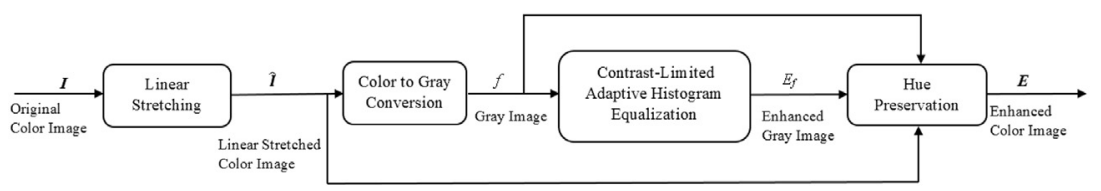

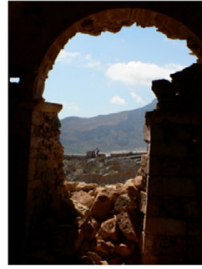

Original Color Image

I

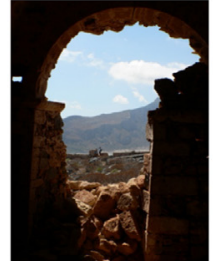

Lincar Stretched Color Image

$\hat{\imath}$

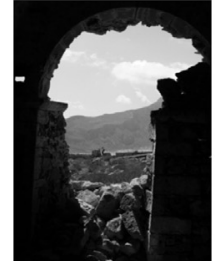

Gray Image

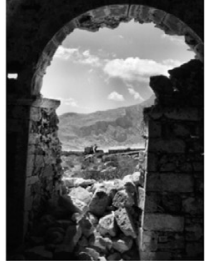

Enhanced Gray Image

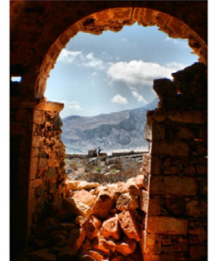

Enhanced Color Image

E

Fig. 4. The overview of the local contrast adaptive enhancement method.

The variational-based fusion model via contrast optimization and color correction is achieved by minimizing the following energy functional:

$$
\begin{aligned}
\mathbf{F}= & \underset{\mathbf{Z}}{\arg \min }\left\{\alpha \int_{\Omega}\left(\omega_{\mathbf{G}(\mathbf{x})}(\mathbf{Z}(\mathbf{x})-\mathbf{G}(\mathbf{x}))^{2}+\omega_{\mathbf{E}(\mathbf{x})}(\mathbf{Z}(\mathbf{x})-\mathbf{E}(\mathbf{x}))^{2}\right) d \mathbf{x}\right. \\
& +\beta \int_{\Omega}\left(\frac{1}{2}(\mathbf{Z}(\mathbf{x})-\hat{\mathbf{G}}(\mathbf{x}))^{2}+\frac{1}{2}(\mathbf{Z}(\mathbf{x})-\hat{\mathbf{E}}(\mathbf{x}))^{2}\right) d \mathbf{x} \\
& \left.-\gamma \int_{\Omega} \int_{\Omega} g_{\sigma}(\mathbf{x}, \mathbf{y}) \Psi_{\epsilon}(\mathbf{Z}(\mathbf{x})-\mathbf{Z}(\mathbf{y})) d \mathbf{x} d \mathbf{y}\right\},
\end{aligned}
$$

where, $\mathbf{G}(\mathbf{x})$ and $\mathbf{E}(\mathbf{x})$ are the globally enhanced image and the locally enhanced image, $\omega_{\mathbf{G}(\mathbf{x})}$ and $\omega_{\mathbf{E}(\mathbf{x})}$ are the corresponding weight maps, and $\hat{\mathbf{G}}(\mathbf{x}), \hat{\mathbf{E}}(\mathbf{x})$ are the corresponding color correction results via midway image equalization [42]. The function $g_{\sigma}(\mathbf{x}, \mathbf{y})$ is a Gaussian curve, where $\mathbf{x}$ and $\mathbf{y}$ denote the positions of two pixels on the image domain. $\Psi_{\epsilon}$ is a non-linear function. $\alpha$, $\beta$ and $\gamma$ are the three parameters that control data fidelity, color consistency, and local contrast.

Data fidelity. The first part of the energy functional (the first line in Eq. (10)) is to achieve data fidelity. This term can be considered as a general fusion framework, which produces a trade-off fusion result between the globally enhanced image $\mathbf{G}(\mathbf{x})$ and the locally enhanced image $\mathbf{E}(\mathbf{x})$. This process provides an attachment to the original data.

The weight maps of the globally enhanced image and the locally enhanced image are computed by

$$
\begin{aligned}
& W_{d}=\min \left\{C_{d}, B_{d}\right\}, \quad d \in\{\mathbf{G}, \mathbf{E}\}, \\
& \omega_{d}=\frac{W_{d}}{W_{\mathbf{G}}+W_{\mathbf{E}}}, \quad d \in\{\mathbf{G}, \mathbf{E}\},
\end{aligned}
$$

where $W_{d}$ is the weight map, $\omega_{d}$ is the normalized weight map, $C_{d}$ is the contrast measure, $B_{d}$ is the brightness measure, and the operation min can efficiently penalize the corresponding lowcontrast, low brightness (under-exposure) or high brightness (overexposure). $C_{d}$ is obtained by a Laplacian filter, which can assign high weights to edges and textures in the corresponding image. $B_{d}$ is computed by a Gaussian curve $\exp \left(-\frac{(i-0.5)^{2}}{2 \sigma_{1}^{2}}\right)$ and $\sigma_{1}=0.2$ in our experiments, which can assign high weights to pixel values close to 0.5 and define low weights to pixel values near 0 (underexposure) or near 1 (over-exposure).

Color consistency. The second part of the energy functional (the second line in Eq. (10)) is to maintain the color consistency. Most previous research $[4,20,21,43]$ considers the gray world principle to achieve the color consistency in image enhancement. Actually, the gray world principle does not always work well for color consistency. We apply color balance [23] as the solution. $\hat{\mathbf{G}}(\mathbf{x})$ and $\hat{\mathbf{E}}(\mathbf{x})$ are the corresponding color correction results of $\mathbf{G}(\mathbf{x})$ and $\mathbf{E}(\mathbf{x})$ after the midway image equalization. $\hat{\mathbf{G}}(\mathbf{x})$ and $\hat{\mathbf{E}}(\mathbf{x})$ share the same cumulative histogram, which makes them have the same color style. The final enhanced result $\mathbf{F}$ can maintain color consistency with $\hat{\mathbf{G}}(\mathbf{x})$ and $\hat{\mathbf{E}}(\mathbf{x})$ via this strategy.

Local contrast improvement. The third part of the energy functional (the third line in Eq. (10)) is to improve the local contrast of the final enhanced result $\mathbf{F}$. The Gaussian function $g_{\sigma}(\mathbf{x}, \mathbf{y})$ is given in Eq. (12).

$g_{\sigma}(\mathbf{x}, \mathbf{y})=\frac{1}{2 \pi \sigma^{2}} \exp \left(\frac{-|\mathbf{x}-\mathbf{y}|^{2}}{2 \sigma^{2}}\right)$,

where $\sigma$ is a parameter representing the size of the Gaussian kernel, and $\mathbf{x}, \mathbf{y}$ denote the positions of two pixels on the image domain. The function $\Psi_{\epsilon}(z)$ is given in Eq. (13).

$$
\Psi_{\epsilon}(z)=\sqrt{z^{2}+\epsilon^{2}},
$$

where $\epsilon$ is a small constant. This equation describes a non-linear function. Its sigmoid-shaped derivation $\Psi_{\epsilon}^{\prime}(z)=\frac{z}{\sqrt{z^{2}+\epsilon^{2}}}$ appearing in Eq. (14) provides the non-linear response of the human visual system, with respect to a contrast enhancement function $[43,44]$.

\subsubsection{Numerical method}

The problem of Eq. (10) can be solved with the gradient descent method. With the iteration index $t$ and the time step $\tau$, the solution is described in Eq. (14).

$$
\begin{aligned}
\mathbf{Z}^{t+1}(\mathbf{x})= & \mathbf{Z}^{t}(\mathbf{x})(1-2(\alpha+\beta) \tau) \\
& +(2 \alpha \mathbf{Q}(\mathbf{x})+2 \beta \hat{\mathbf{Q}}(\mathbf{x}) \\
& \left.+2 \gamma \int_{\Omega} g_{\sigma}(\mathbf{x}, \mathbf{y}) \Psi_{\epsilon}^{\prime}\left(\mathbf{Z}^{t}(\mathbf{x})-\mathbf{Z}^{t}(\mathbf{y})\right) d \mathbf{y}\right) \tau,
\end{aligned}
$$

where $\mathbf{Q}(\mathbf{x})=\omega_{\mathbf{G}}(\mathbf{x}) \cdot \mathbf{G}(\mathbf{x})+\omega_{\mathbf{E}}(\mathbf{x}) \cdot \mathbf{E}(\mathbf{x}), \hat{\mathbf{Q}}(\mathbf{x})=\frac{\mathbf{1}}{\mathbf{2}}(\hat{\mathbf{G}}(\mathbf{x})+\hat{\mathbf{E}}(\mathbf{x}))$, $\mathbf{Z}^{0}(\mathbf{x})=\mathbf{Q}(\mathbf{x})$. After each gradient descent step, we account for a simple constraint that $\mathbf{Z}^{t}(\mathbf{x})$ should be in the pixel value range $[0,255]$. Since there are not many pixel intensities falling outside $[0,255]$, a direct clipping strategy is adopted as the projection operation. Because a linear mapping may decrease the contrast of the enhanced results, we do not use it for projecting pixel values. 
Table 1

The relation between the final iteration stop numbers and the steps $\tau$. The test image $I$ is shown in Fig. 1. The image size is $432 \times 576$.

\begin{tabular}{llllll}
\hline Step $\tau$ & 0.01 & 0.02 & 0.03 & 0.04 & 0.05 \\
\hline Iteration stop number & 96 & 74 & 60 & 50 & 44 \\
\hline
\end{tabular}

\subsubsection{Parameters setting}

The proposed variation-based fusion model produces enhanced images by minimizing the energy functional described in Eq. (10). The solution for this model is given in Eq. (14). There are some parameters that need to be carefully selected. $\alpha, \beta$ and $\gamma$ are the three parameters controlling the data fidelity, the color consistency, and the local contrast. The larger the values, the more influence their terms have in the final enhanced images. In our implementation, we set $\alpha=0.5, \beta=0.5, \gamma=1$ to achieve the balance of data fidelity, color consistency, and local contrast improvement. The function $g_{\sigma}(\mathbf{x}, \mathbf{y})$ is a Gaussian curve given in Eq. (12). The parameter $\sigma$ represents the size of the Gaussian kernel. Smaller values of $\sigma$ increase the local contrast improvement. However, a smaller $\sigma$ might produce unnaturally enhanced results. In order to make a trade-off, we set $\sigma$ to be equal to the $\min ($ high, width)/20. high and width are the height and the width of images, respectively. The function $\Psi_{\epsilon}^{\prime}(z)=\frac{z}{\sqrt{z^{2}+\epsilon^{2}}}$ appearing in Eq. (14) provides the non-linear response to the human visual system, with respect to a contrast enhancement function. The parameter $\epsilon$ is a small constant, which controls the non-linearity. In our implementation, we set $\epsilon=0.1$.

The parameter $\tau$ is the gradient descent step and is a very important parameter impacting the minimization. In order to select a suitable $\tau$ for achieving satisfactorily enhanced results, we give the relation between the final iteration numbers and the values of $\tau$ in Table 1 . The iteration will stop when the mean difference (absolute value) between the previous iteration and the current iteration is less than $\Delta=0.0002$, or the iteration reaches the maximum number Iter $=100$. From Table 1 , we know that smaller $\tau$ results in the need for too many iterations for the convergence. In our implementation with Matlab code, each iteration needs around 8 seconds for the test image $I(432 \times 576)$ shown in Fig. 1 . The computation time can be improved by using other approximation minimization solutions and $\mathrm{C}++$ programming. In our testing, we also know that larger $\tau$ might produce over-enhanced results. In order to achieve a balance between the convergence speed and the naturalness of the enhanced images, we set $\tau=0.02, \Delta=0.001$, Iter $=$ 20 for all of the experiments in the following sections. For the dataset mentioned above, the average iteration stop number is 15 .

\subsection{Fusion with other global enhancement and local enhancement methods}

One of the contributions of this paper is presenting a new method for combining globally enhanced images and locally enhanced images. The global contrast adaptive enhancement method in Section 3.1 is developed from Arici et al.s Histogram Modification Framework (HMF) [5], Wu's tone distortion measure [41], and Nikolova et al.s hue preservation method [40]. The local contrast adaptive enhancement method in Section 3.2 is developed from the traditional CLAHE [16] and Nikolova et al.s hue preservation method [40]. Any other global enhancement method and local enhancement method can replace the method in Section 3.1 and Section 3.2, respectively. For example, Arici et al.'s contrast enhancement method using Histogram Modification Framework (HMF) [5] can replace the global enhancement method in Section 3.1. Banić et al.'s Smart Light Random Mem-

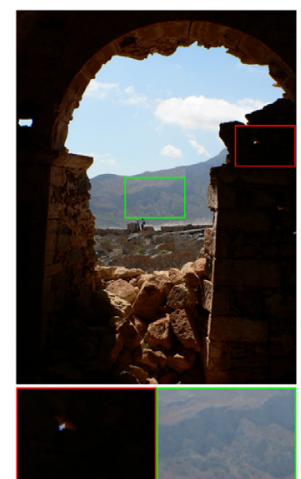

(a)

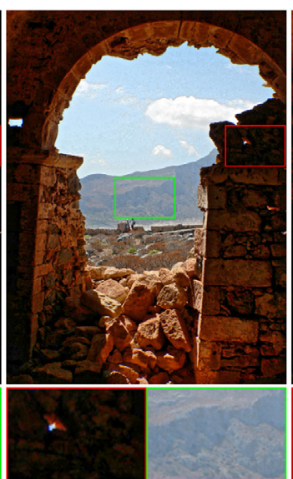

(b)

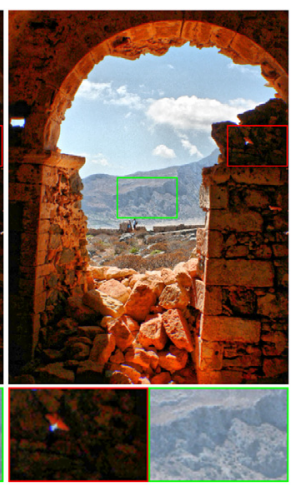

(c)
Fig. 5. Final enhanced results via the fusion model with different global enhancement methods and local enhancement methods. (a). Original image; (b). Fusion with HMF [5] and SLRMSR [6]; (c). Fusion with the method in Section 3.1 and the method in Section 3.2. To allow for visual comparisons, the regions in the red and green rectangles are enlarged and shown below the corresponding images. (For interpretation of the references to color in this figure legend, the reader is referred to the web version of this article.)

ory Sprays Retinex (SLRMSR) [6] can replace the local enhancement method in Section 3.2. The final enhanced results can be produced via the fusion model in Section 3.3. We show results in Fig. 5 in which the proposed method outperforms the compared method in terms of contrast improvement.

\section{Experiments and comparisons}

We ran the proposed method and several state-of-the-art image enhancement methods on a dataset $[45,46]$ including 24 nonuniform illumination color images. The test dataset is a collection of challenging cases for image enhancement. Each image in this dataset has some regions correctly exposed and other regions severely under-exposed or over-exposed. A good enhancement algorithm should enhance the under-exposed regions and the overexposed regions. Meanwhile, the well-exposed regions should not be affected.

The comparison algorithms include Arici et al.'s contrast enhancement method using Histogram Modification Framework (HMF) [5], Banić et al.'s Smart Light Random Memory Sprays Retinex (SLRMSR) [6], Ignatov et al.'s enhancement method with Deep Convolutional Networks (DeepNet) [8], Fu et al.'s Weighted Variational Model for image enhancement (WVM) [47], Ying et al.'s Bio-Inspired Multi-Exposure Fusion framework for image enhancement (BIMEF) [7,38], and Tian et al.'s Global-Local Fusion method for contrast enhancement (GLF) [18]. The source code or executable demos of SLRMSR, DeepNet, WVM, and BIMEF are available on the corresponding project homepages. We reproduced the method HMF with the parameter $\lambda=1$ for all experiments. The code of GLF and the proposed method will be available on the project homepage ${ }^{1}$. The parameter settings of the proposed method are analyzed in Section 3.3.3.

To evaluate the performance of the proposed adaptive enhancement method, both qualitative comparisons and quantitative assessments are considered in the following sections.

\subsection{Qualitative comparisons}

In this section, we show some original test images (nonuniform illumination color images) and the corresponding en-

\footnotetext{
html. 


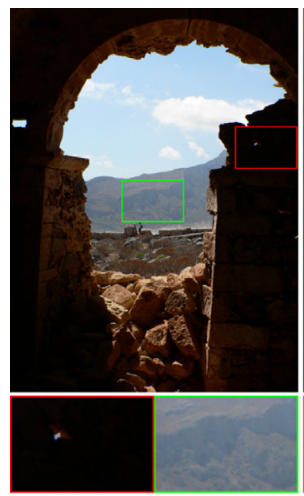

(a) Original

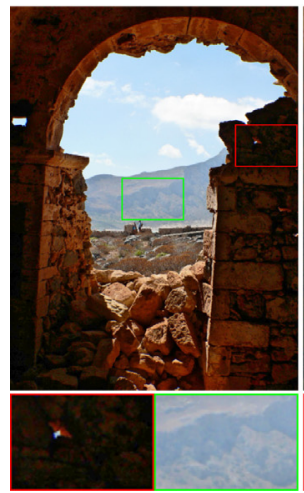

(e) WVM

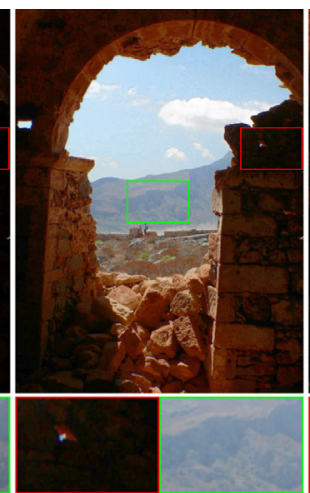

(b) $\mathrm{HMF}$

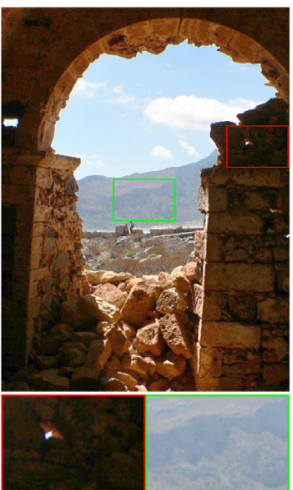

(f) BIMEF

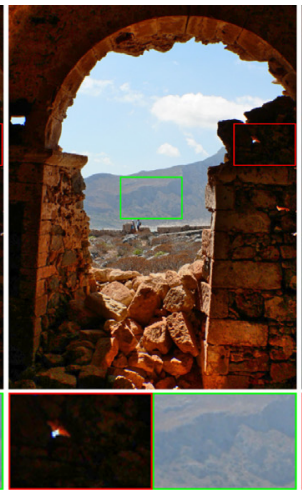

(c) SLRMSR

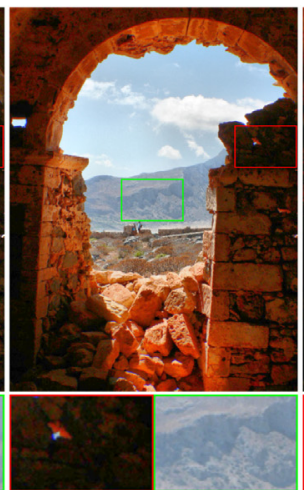

(g) GLF

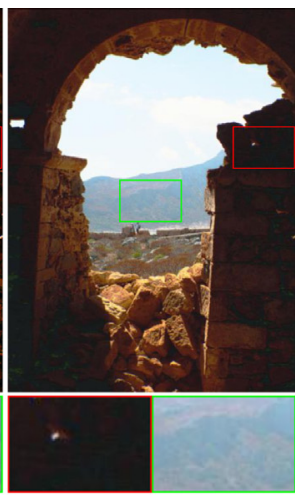

(d) DeepNet

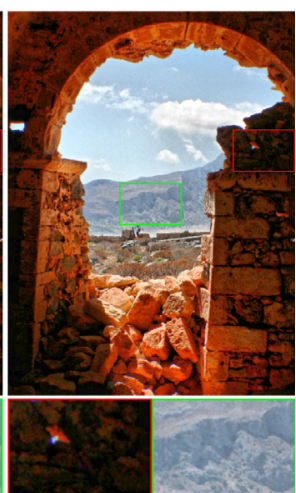

(h) Proposed

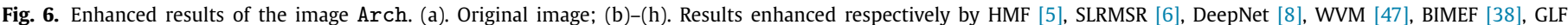

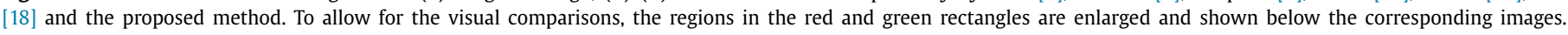
(For interpretation of the references to color in this figure legend, the reader is referred to the web version of this article.)

hanced images to subjectively compare the performance of these enhancement algorithms. Contrast enhancement and detailed information preservation are considered in the comparisons.

Fig. 6 shows the enhanced results on the image Arch (Fig. 4(a)), which contains high illuminations on the sky region and low illuminations on the arch region. Our goal is to enhance the bright regions (high illuminations) and the dark regions (low illuminations), without reducing the details in the bright regions. HMF (Fig. 4(b)) and SLRMSR (Fig. 4(c)) slightly enhances the bright regions and the dark regions. DeepNet (Fig. 4(d)) over-enhances the bright regions (mountain, sky, and so on) and fails in the dark regions. WVM (Fig. 4(e)) over-enhances the bright regions (mountain, sky, and so on) and obtains good results in the dark area. BIMEF (Fig. 4(f)) also over-enhances the bright regions (mountain, sky, and so on) and obtains good results in the dark regions. GLF (Fig. 4(g)) produces good results both in the bright regions and the dark regions. In the bright regions and the dark regions, the proposed method (Fig. 4(h)) obtains desirable results in terms of improving contrasts and preserving details. From the enlarged bright regions, we can observe that the proposed method obtains better contrasts than the results of GLF. Our method generates the best results both in the dark regions and in the bright regions, which is a result of combining the globally enhanced image and the locally enhanced image via contrast optimization and color correction.

Other results are shown in Figs. 7-10, which demonstrate that the proposed method can obtain the best results both in the bright regions and in the dark regions. HMF, SLRMSR, DeepNet, WVM, and BIMEF either slightly enhance the dark regions or over-enhance the bright areas, which produces undesirably enhanced results. GLF can obtain good results both in the bright regions and in the dark regions. However, the results produced by GLF are slightly worse than the proposed method in terms of contrast improvement. The proposed method achieves more local contrast than GFL. Since the differences are not easy to discern in this paper, the visual comparisons are also given on the project homepage.

\subsection{Quantitative comparisons}

For the quantitative evaluation of our method, two widely adopted image quality assessment metrics are considered below. Hautière et al. [48] proposed a contrast evaluation method via Visible Edges Assessment (VEA). This metric evaluates the contrast improvement with three indicators. The first indicator $e$ evaluates the ability for restoring edges. The second indicator $\bar{r}$ is the mean ratio of gradients in visible edges. The third indicator $\Sigma$ represents the percentage of pixels becoming saturated after the enhancement. The higher values of $e$ and $\bar{r}$ indicate better contrast improvements. The lower value of $\Sigma$ indicates the better ability of pixel value range preservation. The evaluation results are shown in Table 2, which demonstrates that the proposed method outperforms other algorithms in terms of contrast improvement (higher $e$ and $\bar{r}$ ). The contrast optimization of the proposed method can generate more visible edges. For the pixel value range preservation (lower $\Sigma$ ), DeepNet obtains the best result. The proposed method does not achieve desirable performance $\Sigma$ since the simple clipping projection for pixels falling outside $[0,255]$ is adopted in the variational iteration. However, the performance of $\Sigma$ has little effect on the image visual quality improvement. As shown in Figs. 6-9, the proposed method achieves the best overall results even though the performance of $\Sigma$ is not the best.

Panetta et al. [49] proposed a color image quality measurement (CQE) that combines the contrast, sharpness and colorfulness met- 


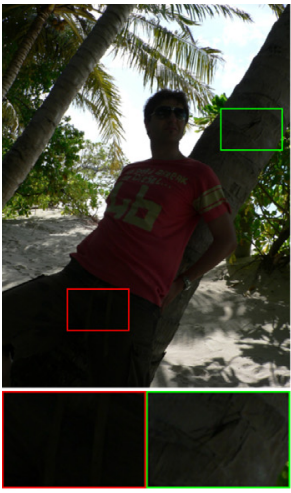

(a) Original

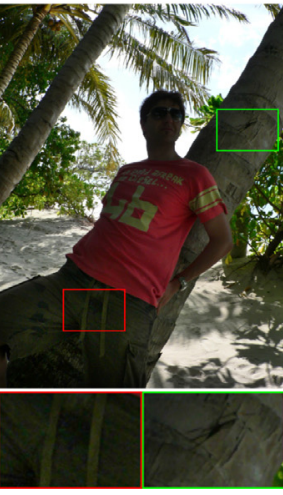

(e) WVM

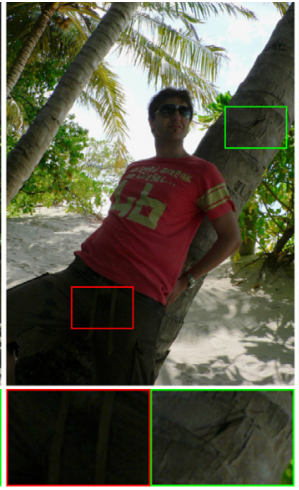

(b) $\mathrm{HMF}$

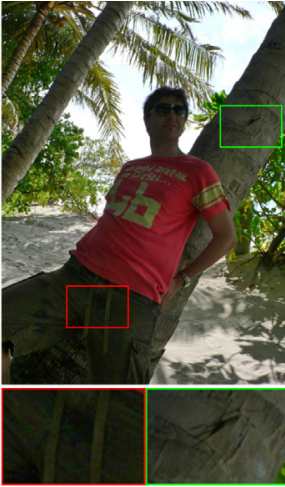

(c) SLRMSR

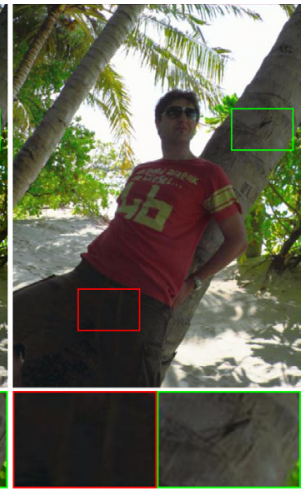

(d) DeepNet

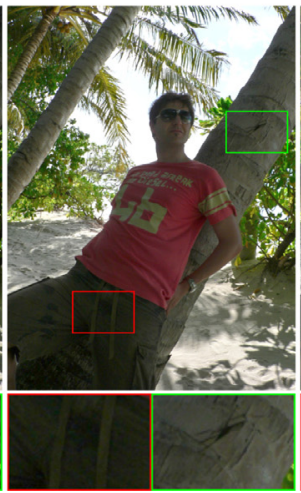

(f) BIMEF

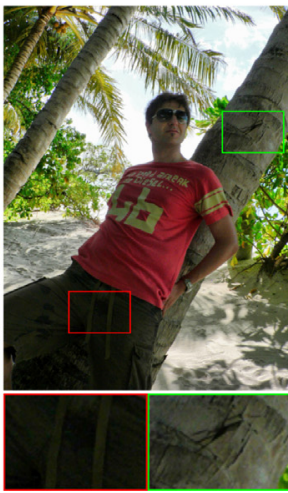

(g) GLF

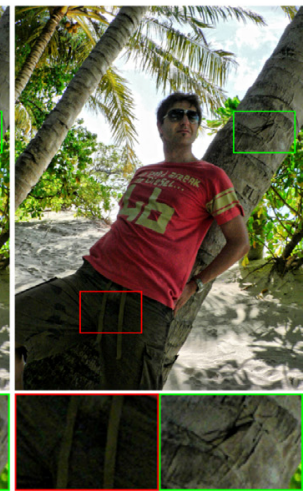

(h) Proposed

Fig. 7. Enhanced results of the image Man1. (a). Original image; (b)-(h). Results enhanced respectively by HMF [5], SLRMSR [6], DeepNet [8], WVM [47], BIMEF [38], GLF [18] and the proposed method.

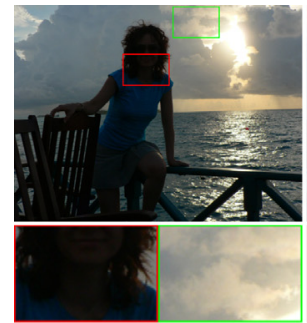

(a) Original

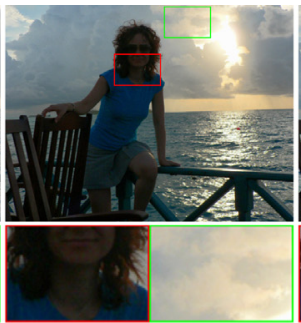

(b) HMF

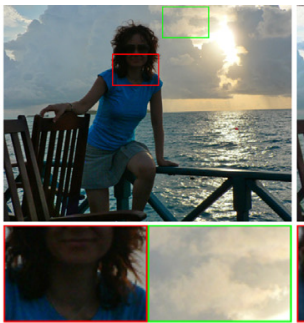

(c) SLRMSR

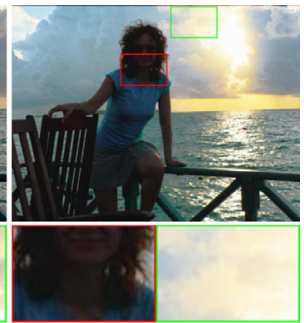

(d) DeepNet

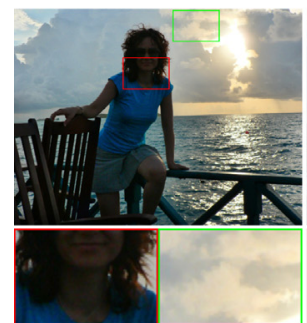

(e) WVM

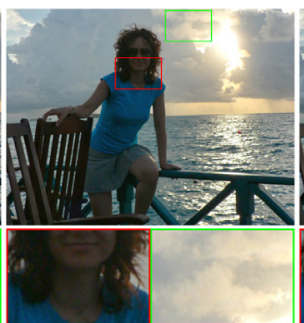

(f) BIMEF

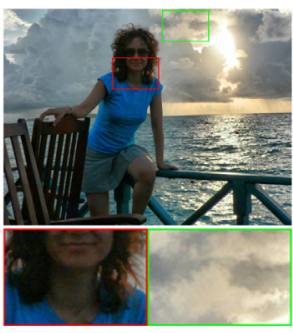

(g) GLF

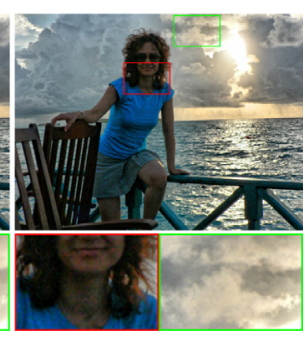

(h) Proposed

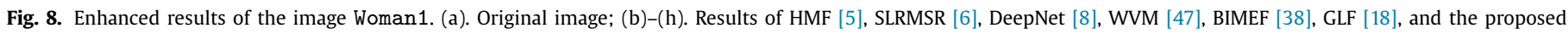
method, respectively.

Table 2

Average VEA of enhancement algorithms on the test dataset.

\begin{tabular}{llllllll}
\hline & HMF [5] & SLRMSR [6] & DeepNet [8] & WVM [47] & BIMEF [38] & GLF [18] & Proposed \\
\hline$e$ & 0.1858 & 0.2064 & -0.0497 & 0.1965 & 0.1494 & 0.2482 & $\mathbf{0 . 3 5 9 7}$ \\
$\bar{r}$ & 1.9781 & 2.3539 & 1.4993 & 2.0218 & 1.9950 & 2.4277 & $\mathbf{3 . 0 4 8 3}$ \\
$\Sigma(\%)$ & 0.0046 & 0.0336 & $\mathbf{0}$ & 0.0513 & 0.0077 & 0.0137 & 0.2116 \\
\hline
\end{tabular}




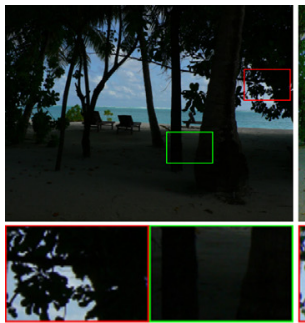

(a) Original

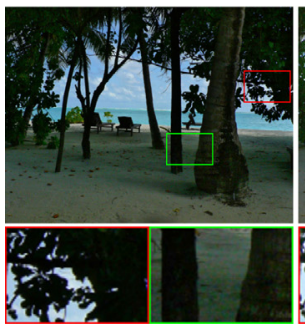

(e) WVM

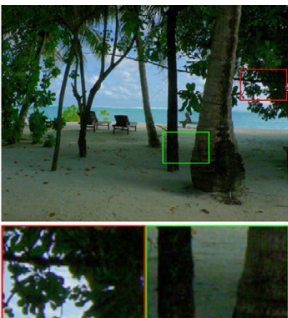

(b) HMF

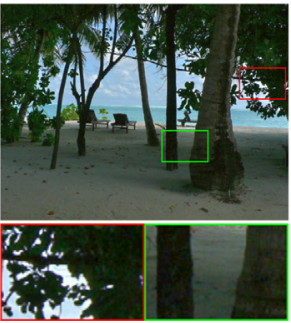

(f) BIMEF

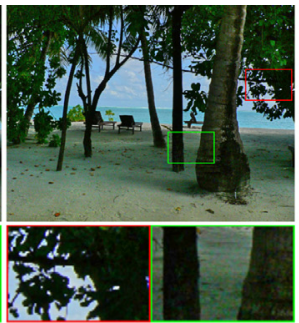

(c) SLRMSR

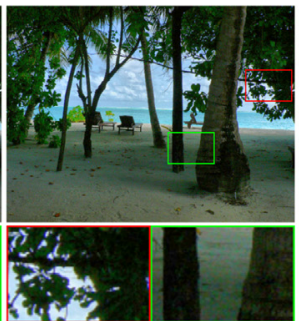

(g) GLF

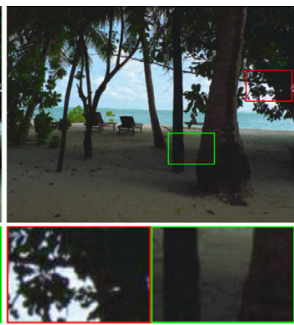

(d) DeepNet

Fig. 9. Enhanced results of the image Beach. (a). Original image; (b)-(h). Results enhanced respectively by HMF [5], SLRMSR [6], DeepNet [8], WVM [47], BIMEF [38], GLF [18], and the proposed method.

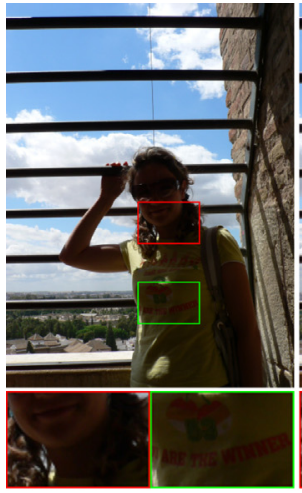

(a) Original

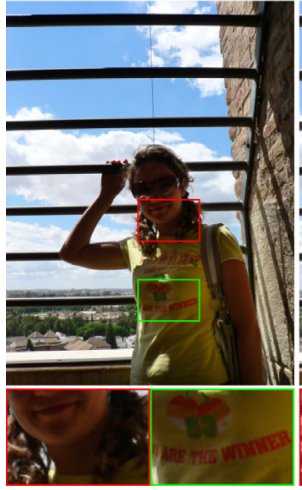

(e) WVM

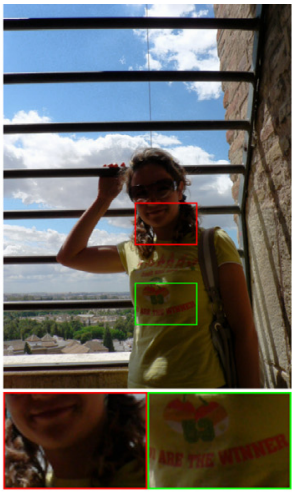

(b) HMF

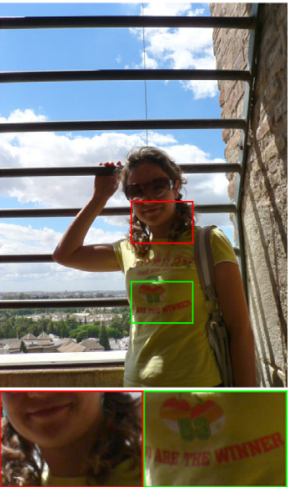

(f) BIMEF

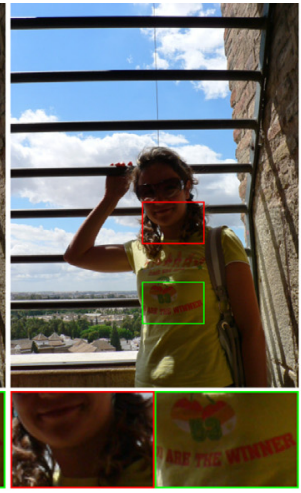

(c) SLRMSR

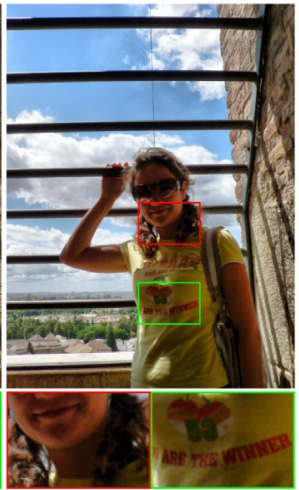

(g) GLF

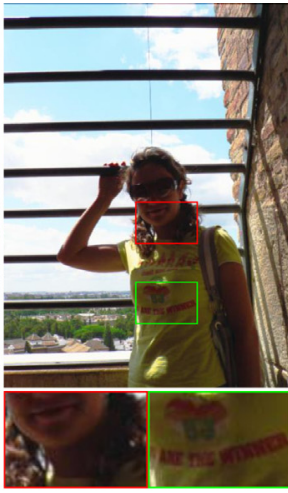

(d) DeepNet

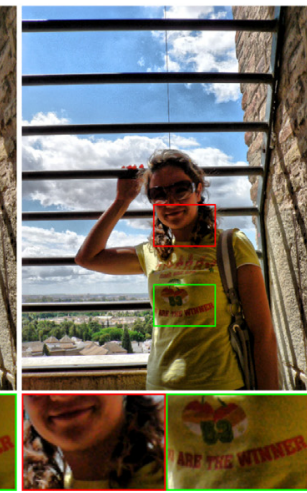

(h) Proposed

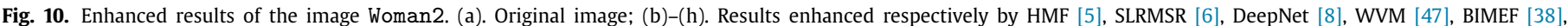
GLF [18] and the proposed method.

rics. CQE is the weighted sum of the three metrics. The contrast is represented by the Michelson-Law measure of enhancement AME [50] of the intensity component. The sharpness is represented by the Weber contrast based measure of enhancement EME [51] of each gray edge map. The colorfulness is represented with the statistical information [52] of the opponent color components. The higher the value of CQE, the better is the quality of the considered color image. We use these metrics to evaluate our method in comparison to several algorithms on the test dataset. The results shown in Table 3 indicate that the proposed method outperforms other algorithms in terms of image quality. The contrast optimization in the variational-based fusion model ensures that our method 
Table 3

Average CQE of the enhanced images.

\begin{tabular}{llllllll}
\hline & HMF [5] & SLRMSR [6] & DeepNet [8] & WVM [47] & BIMEF [38] & GLF [18] & Proposed \\
\hline CQE & 0.4601 & 0.4926 & 0.3970 & 0.4629 & 0.4504 & 0.5052 & $\mathbf{0 . 5 7 5 5}$ \\
Contrast & 0.3510 & 0.3826 & 0.2326 & 0.3757 & 0.3234 & 0.3595 & $\mathbf{0 . 5 3 0 4}$ \\
Sharpness & 0.5981 & 0.6373 & 0.5589 & 0.5909 & 0.6231 & 0.6776 & $\mathbf{0 . 7 0 0 9}$ \\
Colorfulness & 0.4290 & 0.4547 & 0.4049 & 0.4170 & 0.4001 & 0.4779 & $\mathbf{0 . 4 8 1 9}$ \\
\hline
\end{tabular}

achieves better results with higher contrast and sharpness. The hue preservation considered in enhancements and the color correction considered in the variational-based fusion give our method the ability to achieve desirable colorfulness.

\section{Conclusions}

In this paper, we have proposed a variational-based fusion method for non-uniform illumination image enhancement via contrast optimization and color correction. The main contributions of our paper are as follows. First, a global contrast adaptive enhancement method is introduced to improve the global contrast. Second, a hue preservation framework is considered in the global enhancement and the local enhancement. Third, a variational-based image fusion method is developed for obtaining final enhanced images. Experiments demonstrate that our proposed method outperforms other enhancement methods in terms of subject visual comparisons and objective performance evaluations.

Since there is undesirable noise in the dark regions of some non-uniform illumination images, noise reduction will be considered in the contrast enhancement framework in the future work. The numerical method used to solve the minimization problem is highly complex and requires more time to achieve satisfactory results. We will consider other approximate minimization methods to complete this task in the future.

\section{Acknowledgments}

We would like to sincerely thank Vassilios Vonikakis for sharing the test images and permitting us to use their images in this paper. Many thanks to Nikola Banić, Nikolay Kobyshev, Xueyang Fu, Zhenqiang Ying, and Nicolas Hautière for sharing their code or demos for experiment comparisons. We greatly thank the anonymous reviewers for their helpful comments that allowed us to improve this work.

\section{References}

[1] K. Zuiderveld, Contrast limited adaptive histogram equalization, in: Graphics Gems IV, Academic Press Professional, Inc., San Diego, CA, USA, 1994, pp. 474-485.

[2] J.A. Stark, Adaptive image contrast enhancement using generalizations of histogram equalization, IEEE Trans. Image Process. 9 (5) (2000) 889-896.

[3] S.K. Naik, C. Murthy, Hue-preserving color image enhancement without gamut problem, IEEE Trans. Image Process. 12 (12) (2003) 1591-1598.

[4] E. Provenzi, C. Gatta, M. Fierro, A. Rizzi, A spatially variant white-patch and gray-world method for color image enhancement driven by local contrast, IEEE Trans. Pattern Anal. Mach. Intell. 30 (10) (2008) 1757-1770.

[5] T. Arici, S. Dikbas, Y. Altunbasak, A histogram modification framework and its application for image contrast enhancement, IEEE Trans. Image Process. 18 (9) (2009) 1921-1935

[6] N. Banić, S. Lončarić, Smart light random memory sprays retinex: a fast retinex implementation for high-quality brightness adjustment and color correction, JOSA A 32 (11) (2015) 2136-2147.

[7] Z. Ying, G. Li, Y. Ren, R. Wang, W. Wang, A new image contrast enhancement algorithm using exposure fusion framework, in: 17th International Conference on Computer Analysis of Images and Patterns (CAIP), Springer, 2017, pp. 36-46.

[8] A. Ignatov, N. Kobyshev, R. Timofte, K. Vanhoey, L. Van Gool, DSLR-Quality photos on mobile devices with deep convolutional networks, in: IEEE International Conference on Computer Vision (ICCV), 2017, pp. 3277-3285.

[9] L. Yuan, J. Sun, Automatic exposure correction of consumer photographs, in: European Conference on Computer Vision (ECCV), Springer, 2012, pp. 771-785.

[10] W.-Y. Hsu, C.-Y. Chou, Medical image enhancement using modified color histogram equalization, J. Med. Biol. Eng. 35 (5) (2015) 580-584.
[11] J.-L. Lisani, J. Michel, J.-M. Morel, A.B. Petro, C. Sbert, An inquiry on contrast enhancement methods for satellite images, IEEE Trans. Geosci. Remote Sens. 54 (12) (2016) 7044-7054.

[12] G. Schaefer, M.I. Rajab, M.E. Celebi, H. Iyatomi, Colour and contrast enhancement for improved skin lesion segmentation, Comput. Med. Imaging Graph. 35 (2) (2011) 99-104.

[13] S.-W. Kim, J.-Y. Jung, C.-H. Yoo, S.-J. Ko, Retinex-based illumination normalization using class-based illumination subspace for robust face recognition, Signal Process. 120 (2016) 348-358.

[14] E. Reinhard, W. Heidrich, P. Debevec, S. Pattanaik, G. Ward, K. Myszkowski, High Dynamic Range Imaging: Acquisition, Display, and Image-based Lighting, Morgan Kaufmann, 2010.

[15] R.C. Gonzalez, R.E. Woods, Digital Image Processing, 3rd ed., Prentice-Hall, Englewood Cliffs, NJ, USA, 2007.

[16] S.M. Pizer, E.P. Amburn, J.D. Austin, R. Cromartie, A. Geselowitz, T. Greer, B. ter Haar Romeny, J.B. Zimmerman, K. Zuiderveld, Adaptive histogram equalization and its variations, Comput. Vision Graph. Image Process. 39 (3) (1987) 355-368

[17] T. Mertens, J. Kautz, F. Van Reeth, Exposure fusion: a simple and practical alternative to high dynamic range photography, Comput. Graph. Forum 28 (1) (2009) 161-171.

[18] Q.-C. Tian, L.D. Cohen, Global and local contrast adaptive enhancement for non-uniform illumination color images, in: IEEE International Conference on Computer Vision Workshops (ICCV Color and Photometry in Computer Vision Workshop), IEEE, 2017, pp. 3023-3030.

[19] E. Provenzi, Computational Color Science: Variational Retinex-like Methods, John Wiley \& Sons, 2017.

[20] R. Palma-Amestoy, E. Provenzi, M. Bertalmío, V. Caselles, A perceptually inspired variational framework for color enhancement, IEEE Trans. Pattern Anal. Mach. Intell. 31 (3) (2009) 458-474.

[21] F. Pierre, J.-F. Aujol, A. Bugeau, G. Steidl, V.-T Ta, Variational contrast enhancement of gray-scale and RGB images, J. Math. Imaging Vis. 57 (1) (2017) 99-116.

[22] Q.-C. Tian, L.D. Cohen, Histogram-based color transfer for image stitching, J. Imaging 3 (3) (2017) 38.

[23] Q.-C. Tian, L.D. Cohen, Color consistency for photo collections without gamut problems, in: the 23rd International Conference on Multimedia Modeling (MMM), Springer, 2017, pp. 90-101.

[24] Q-C. Tian, L.D. Cohen, Naturalness preservation image contrast enhancement via histogram modification, in: Proc. SPIE 10615, Ninth International Conference on Graphic and Image Processing (ICGIP 2017), SPIE, 2018, p. 106152 U.

[25] V. Caselles, J.-L. Lisani, J.-M. Morel, G. Sapiro, Shape preserving local histogram modification, IEEE Trans. Image Process. 8 (2) (1999) 220-230.

[26] K. Sim, C. Tso, Y. Tan, Recursive sub-image histogram equalization applied to gray scale images, Pattern Recognit. Lett. 28 (10) (2007) 1209-1221.

[27] A.R. Rivera, B. Ryu, O. Chae, Content-aware dark image enhancement through channel division, IEEE Trans. Image Process. 21 (9) (2012) 3967-3980.

[28] H.-D. Liu, M. Yang, Y. Gao, L. Cao, Fast local histogram specification, IEEE Trans. Circuits Syst. Video Technol. 24 (11) (2014) 1833-1843.

[29] E.H. Land, J.J. McCann, Lightness and retinex theory, J. Opt. Soc. Am. 61 (1) (1971) 1-11.

[30] D.J. Jobson, Z.-u. Rahman, G.A. Woodell, A multiscale retinex for bridging the gap between color images and the human observation of scenes, IEEE Trans. Image Process. 6 (7) (1997) 965-976.

[31] A.B. Petro, C. Sbert, J.-M. Morel, Multiscale retinex, Image Process. Line (2014) $71-88$

[32] R. Kimmel, M. Elad, D. Shaked, R. Keshet, I. Sobel, A variational framework for retinex, Int. J. Comput. Vis. 52 (1) (2003) 7-23.

[33] J.-M. Morel, A.B. Petro, C. Sbert, What is the right center/surround for retinex? in: IEEE International Conference on Image Processing (ICIP), IEEE, 2014, pp. $4552-4556$

[34] R.S. Blum, Z. Liu, Multi-Sensor Image Fusion and Its Applications, CRC press, 2005 .

[35] K. Ma, H. Li, H. Yong, Z. Wang, D. Meng, L. Zhang, Robust multi-exposure image fusion: a structural patch decomposition approach, IEEE Trans. Image Process. 26 (5) (2017) 2519-2532.

[36] W.-W. Wang, P.-L. Shui, X.-C. Feng, Variational models for fusion and denoising of multifocus images, IEEE Signal Process. Lett. 15 (2008) 65-68.

[37] X. Fu, D. Zeng, Y. Huang, Y. Liao, X. Ding, J. Paisley, A fusion-based enhancing method for weakly illuminated images, Signal Process. 129 (2016) 82-96.

[38] Z. Ying, G. Li, W. Gao, A bio-inspired multi-exposure fusion framework for lowlight image enhancement, arXiv:1711.00591 (2017).

[39] C.O. Ancuti, C. Ancuti, P. Bekaert, Enhancing by saliency-guided decolorization, in: IEEE Conference on Computer Vision and Pattern Recognition (CVPR), IEEE, 2011, pp. 257-264. 
[40] M. Nikolova, G. Steidl, Fast hue and range preserving histogram specification: theory and new algorithms for color image enhancement, IEEE Trans. Image Process. 23 (9) (2014) 4087-4100.

[41] X. Wu, A linear programming approach for optimal contrast-tone mapping, IEEE Trans. Image Process. 20 (5) (2011) 1262-1272.

[42] J. Delon, Midway image equalization, J. Math. Imaging Vis. 21 (2) (2004) 119-134.

[43] M. Bertalmío, V. Caselles, E. Provenzi, A. Rizzi, Perceptual color correction through variational techniques, IEEE Trans. Image Process. 16 (4) (2007) 1058-1072.

[44] J.J. McCann, The role of simple nonlinear operations in modeling human lightness and color sensations, in: Human Vision, Visual Processing, and Digital Display, 1077, International Society for Optics and Photonics, 1989, pp. 355-364.

[45] V. Vonikakis, I. Andreadis, A. Gasteratos, Fast centre-surround contrast modification, IET Image Proc. 2 (1) (2008) 19-34.

[46] V. Vonikakis, A collection of the most challenging cases for image enhancement, Accessed on 02-January-2018, (https://sites.google.com/site/vonikakis/ datasets/challenging-dataset-for-enhancement).

[47] X. Fu, D. Zeng, Y. Huang, X.-P. Zhang, X. Ding, A weighted variational model for simultaneous reflectance and illumination estimation, in: IEEE Conference on Computer Vision and Pattern Recognition (CVPR), 2016, pp. 2782-2790.

[48] N. Hautière, J.-P. Tarel, D. Aubert, E. Dumont, Blind contrast enhancement assessment by gradient ratioing at visible edges, Image Anal. Stereol. 27 (2) (2011) 87-95.

[49] K. Panetta, C. Gao, S. Agaian, No reference color image contrast and quality measures, IEEE Trans. Consum. Electron. 59 (3) (2013) 643-651.

[50] S.S. Agaian, B. Silver, K.A. Panetta, Transform coefficient histogram-based image enhancement algorithms using contrast entropy, IEEE Trans. Image Process. 16 (3) (2007) 741-758.

[51] S.S. Agaian, K. Panetta, A.M. Grigoryan, A new measure of image enhancement, in: IASTED International Conference on Signal Processing \& Communication, 2000, pp. 19-22.

[52] D. Hasler, S.E. Suesstrunk, Measuring colorfulness in natural images, in: $\mathrm{Hu}-$ man vision and electronic imaging VIII, 5007, International Society for Optics and Photonics, 2003, pp. 87-96.
Qi-Chong Tian is a Ph.D. candidate at the Center for Research in Decision Mathematics (CEREMADE), the French National Center for Scientific Research (CNRS), Université Paris Dauphine, PSL Research University, Paris, France. He received a M.S. Degree from Department of Electronics and Information Engineering, Huazhong University of Science and Technology in 2013, and received a B.S. Degree from School of Computer Science, China University of Mining and Technology in 2010. He is a technical program committee member of some international conferences. He reviewed manuscripts for more than ten international journals and conferences. His research interests include image enhancement, color correction, and minimization methods.

Laurent D. Cohen is a CNRS Research Director (Directeur de Recherche) at the Center for Research in Decision Mathematics (CEREMADE), the French National Center for Scientific Research (CNRS), Université Paris Dauphine, PSL Research University, Paris, France. He received the Master's Degree and the Ph.D. Degree in Applied Mathematics from University of Paris VI in 1983 and 1986, respectively. He was a student at the École Normale Supérieure Paris from 1981 to 1985. From 1985 to 1987, he was a member of the Computer Graphics and Image Processing Group at Schlumberger Palo Alto and Montrouge Research. He worked with the French Institute for Research in Computer Science and Automation (INRIA) from 1988 to 1989. Since 1990, he has been a researcher (Chargé de Recherche then Directeur de Recherche 1st class) with CNRS in the Applied Mathematics and Image Processing Group at CEREMADE, Université Paris Dauphine, Paris, France. His research interests include applications of Partial Differential Equations and Variational Methods to Image Processing and Computer Vision, such as deformable models, minimal paths, geodesic curves, surface reconstruction, image segmentation, registration, and restoration. He served as an editorial member of the Journal of Mathematical Imaging and Vision, Medical Image Analysis, Machine Vision and Applications, and Journal of Visual Communication and Image Representation. He was also a program committee member of about 50 international conferences. He authored over 260 publications with more than 12,000 citations (Google Scholar), and hold 5 patents. He received the CS 2002 prize for Signal and Image Processing, the Taylor \& Francis Prize 2006, the 2009 EADS grand prize of the French Academy of Sciences. He was elected as an IEEE Fellow in 2010. 